\title{
Evaluation of Antioxidant Capacity and Flavor Profile Change of Pineapple Peel Vinegar during Fermentation
}

\author{
Liao Liangkun, Huang Hui, Yuan Yuan, Wei Xiaoyi, Lin Lijing, Gong Xiao, Zhang Suhui \\ Agricultural Products Processing Research Institute, Chinese Academy of Tropical Agricultural \\ Sciences, Zhanjiang, 524001, China
}

Keywords: pineapple peel; fermentation; fruit vinegar; antionxidative

Abstract: The study of fermentation process of pineapple peel fruit vinegar analyzed the change of acidity, $\mathrm{pH}$, alcohol content, organic acids and volatile components during fermentation, and revealed its fermentation characteristics. The study of antionxidative activity in the process of fermentation, detected its antionxidative activity change. In the fermentation process, acidity and $\mathrm{pH}$ value in 3 - $6 \mathrm{~d}$ change quickly, also, alcohol in 3 - 6 d drop quickly; The content of acetic acid increased remarkable and in 3 - 6 d rise faster, however the other main organic acids remain stable such as citric acid, malic acid, et al. The volatile substances change considerably after fermentation, and the main component were esters and alcohols before fermentation and this turned into acids and alcohols after fermentation. The antionxidative activity had larger improvement after fermentation. This study will provide technical guidance for fruit vinegar fermentation.

\section{Introduction}

Fruit vinegar is a fruit or its scraps as raw materials, use of vinegar bacteria made from a kind of rich nutrition, unique flavor drink. Research shows that fruit vinegar have fall hematic fat, fall blood pressure, lower cholesterol, protect the cardiovascular [1].At the same time, the fruit vinegar can remove superoxide free radicals, anti-cancer anti-cancer, anti-aging efficacy [2].Fruit vinegar rich in a variety of organic acids and amino acids of human body, can promote metabolism, regulate acid-base balance, eliminate fatigue.

Pineapple is one of the main tropical fruits in China, and its output reached 2.5 million tons; Pineapple postharvest difficult to storage, however, usually only a week or so [3].Economic losses caused by the corruption of pineapple fruit growers in China more than 200 million yuan, therefore, increase the pineapple products deep processing of pineapple industry stable development is particularly important. This research use pineapple peel slag pineapple fruit vinegar production, studies its features in the fermentation process and function change, which will provide a scientific basis for production of high quality of pineapple fruit vinegar. 


\section{Materials and Methods}

\subsection{Chemicals and reagents}

The Bacillus aceticus As1.01 was purchased form China Center of Industrial Culture Collection, All chemicals and reagents used were HPLC grade and obtained from Sinopharm Chemical Reagent Co.,Ltd.(Shanghai, China )

\subsection{Sample preparation}

The fresh pineapple peel which were the residue after juicing donated by a local beverage company (Zhongre technology Co., Ltd Zhanjiang, China). The same quality of water was add to the pineapple peel and adjusted to $18^{\mathrm{O}}$ Brix with sugar before sterilization, the pasteuring for 30 min. The material was inoculated with $1 \%$ activated yeast after cooling, and then fermentation was started and temperature was kept at $28^{\circ} \mathrm{C}$ throughout the fermentation process. After the alcoholic strength no longer increase, started a secondary fermentation by inoculated with 3\% activated Bacillus aceticus and incubation at $30^{\circ} \mathrm{C}$. Vinegar samples were prepared every day and stored and $-20^{\circ} \mathrm{C}$.

\subsection{Determination of quality parameters}

The $\mathrm{PH}$ was measured using a Portable $\mathrm{pH}$-meter, Total acid was detected by titrating with $0.1 \mathrm{~mol} / \mathrm{L} \mathrm{NaoH}$ and expressed as \% acetic acid. Alcohol content (\%) was determined using a pycnometer.

\subsection{Organic acids analysis}

Organic acids were measured using a Shimadzu LC-15C system equipped with degasser, quaternary pump, autosampler, ZORBAXSB-C18 chromatographic column (4.6 250 mm; Agilent, USA) and a SPD-15C UV-detector (Shimadzu, Japan) (Berenguer et al., 2016). Briefly, all samples were centrifuged for 5 min at 15000g (model H2050R-1, Hettich Zentrifugen, Chengdu, China) and then the supernatant was filtered through a $0.22 \mathrm{~lm}$ PVDF membrane. The mobile phase for isocratic elution consisted of $0.1 \%$ phosphoric acid solution with a flow rate of $0.7 \mathrm{~mL} / \mathrm{min}$, loading volume of $10 \mathrm{lL}$ and ultraviolet detector's wavelength of $210 \mathrm{~nm}$. The qualitative and quantitative characterization of citric, malic, tartaric, oxalic and lactic acids were based on the standards and the related chromatographic peaks area[4].

\subsection{Determination of polyphenol and antioxidant activities}

\subsubsection{Determination of total phenolic content}

Total phenols content (TPC) was determined by the Folin- Ciocalteu colorimetric method. Samples $(0.5 \mathrm{~mL}$ of $)$ were diluted 10 times and mixed with $1 \mathrm{~mL}$ of the Folin-Ciocalteu reagent and $2 \mathrm{~mL} \mathrm{7.5 \%} \mathrm{sodium} \mathrm{carbonate.} \mathrm{The} \mathrm{mixture} \mathrm{was} \mathrm{vortexed} \mathrm{and} \mathrm{allowed} \mathrm{to} \mathrm{stand} \mathrm{for} 5 \mathrm{~min}$ at 50C. The absorbance was measured with a spectrophotometer at $760 \mathrm{~nm}$. Gallic acid was used to construct a calibration curve and expressed as gallic acid equivalent (GAE)[5].

\subsubsection{DPPH antioxidant capacity assay}

DPPH radical scavenging activity was determined as described by . The diluted sample (2 mL) 
was added to $0.1 \mathrm{mM}$ DPPH in ethanol (2 mL) and then vortexed. The absorbance was measured at $517 \mathrm{~nm}$ after $30 \mathrm{~min}$ of incubation in dark[6]. Percent DPPH scavenging activity was calculated using the following equation:

\subsubsection{Hydroxyl radicals scavenging activity}

The hydroxyl radicals $(\mathrm{OH})$ were carried out according to literature [7]. The radical inhibition was calculated by the following equation:

Inhibition

where Ao represents the absorbance of control and As represents the absorbance of sample.

\subsection{HS-SPME-GC-MS analysis}

Qualitative analysis of volatiles was performed on a GC-MS QP2010 Series (Shimadzu USA MANUFACTURING, Inc., Kyoto, Japan) as described in previous literature with some modification(Andreu-Sevilla, Mena, Martí, García Viguera, \& Carbonell- Barrachina, 2013). Briefly, volatile compounds were extracted from pomegranate wines, using headspace solid phase micro-extraction (HS-SPME)[8]. $5 \mathrm{~mL}$ of pomegranate wine was placed into a $20 \mathrm{~mL}$ glass vial. Ten times diluted 2-octanol (0.2 lL) was subsequently put into the vial as internal standard (IS). $\mathrm{NaCl}(15 \%)$ was added to inhibit enzymatic degradation and facilitate the evolution of volatiles into the headspace. The vials were sealed immediately by polypropylene cap with PTFE/silicone septa. The volume ratio wine to headspace was 1:3. Glass vials were equilibrated for $20 \mathrm{~min}$ at $45 \mathrm{C}$ in the water bath and then a 50/30 lm DVB/CAR/ PDMS fiber was exposed to the HS for 50 min at $45 \mathrm{C}$ to collect the analytes. The volatile compounds were identified by searching against the NIST 14 library of mass spectral data and comparison of the fragmentation patterns with some literatures previously reported. The relative content of each compound presented here was expressed as\% of total peak areas of all identified compounds. The peaks of 2-octanol in GC-MS total ion chromatogram of all determining samples were kept uniformity to ensure the device and the protocol are stable and the results are reproducable.

\section{Resullts and Discussion}

\subsection{Quality parameters during vinegar making}

The figure 1 shows that the fermentation process of $\mathrm{pH}$ gradually decreases with increasing fermentation experiments, the corresponding acidity rise gradually, in $0 \sim 4 \mathrm{~d}$, fermentation is relatively slow, $\mathrm{pH}$ change is slow, slower, acetic acid accumulation; In $5 \sim 9 \mathrm{~d}, \mathrm{pH}$ and acidity changes faster, higher acidity obviously accelerated, ethanol speed vinegar bacteria oxidation, acetic acid accumulation increased rapidly;9 $12 \mathrm{~d}$, fermentation speed slow, $\mathrm{pH}$ is in the gentle change, acidity change is slow, slow accumulation acetate[9] 


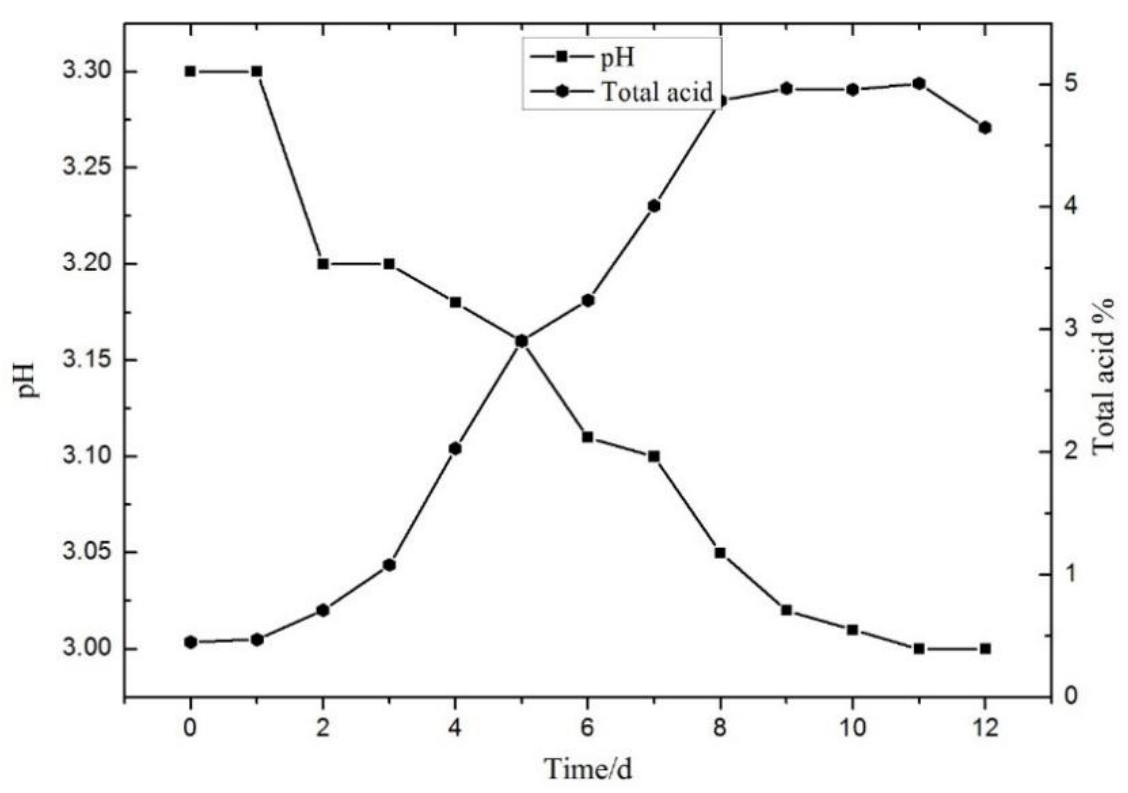

Fig.1 Change of total acid and $\mathrm{pH}$

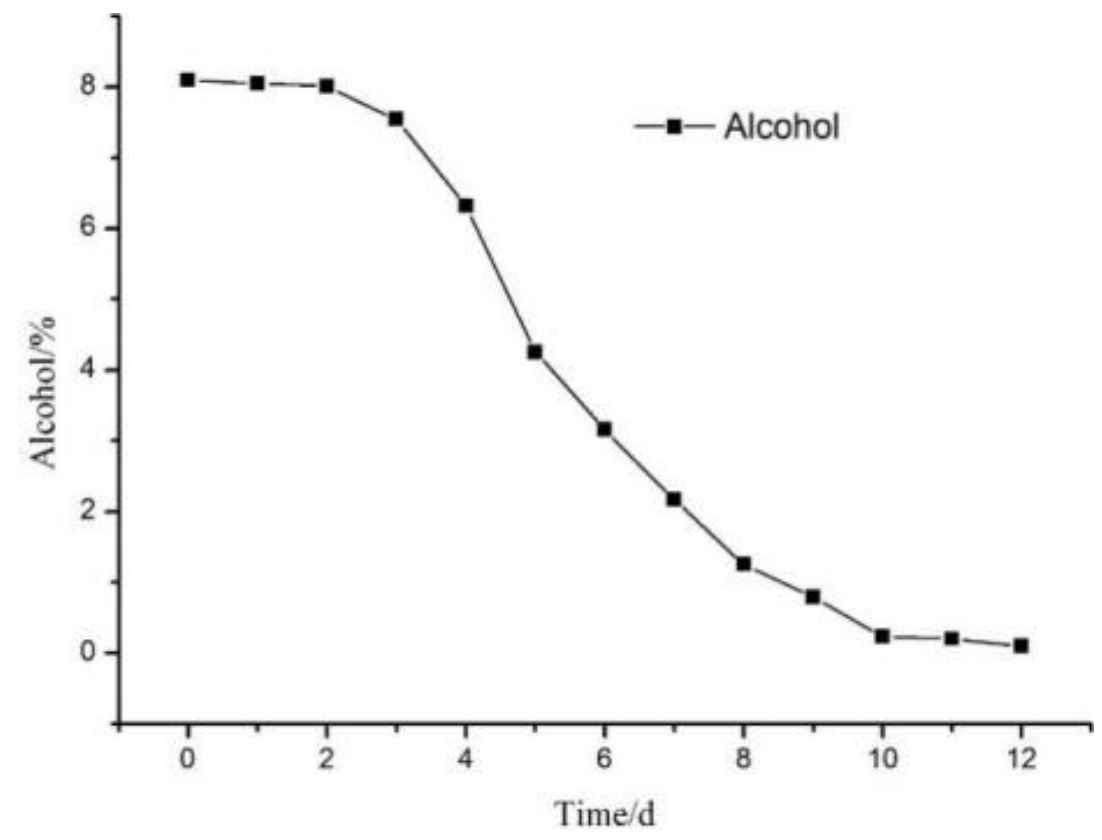

Fig.2 Change of alcohol

The figure 2 shows that in the process of fermentation alcohol acidity present the opposite trend, with the change of the acetic acid fermentation process is a process of ethanol into acetic acid bacilli oxidation, therefore, in the fermentation system, ethanol and acetic total amount remains the same, but the content change each other instead. The acidity and alcohol changes trend shows that in the fermentation period of $3 \sim 6 \mathrm{~d}$, vinegar bacteria activity is higher, oxidation of alcohol acetate production speed quickly, so in this period of time, to guarantee adequate oxygen to complete the oxidation of ethanol .

\subsection{Organic acids during vinegar making}

The figure 3 shows that eight kinds of organic acid standard degree of separation is good, and good linear relationship (R2 > 0.998), so this method analysis of organic acids in the samples are 
available, and the figure 4 shows that in the process of fermentation, in addition to acetic acid content change is bigger, other organic acids content in the relatively stable state.Acetic acid content in $3 \sim 6 \mathrm{~d}$ increases quickly, after $6 \mathrm{~d}$, leveling off, the corresponding changes in acidity and alcohol.By figure 4 shows, the pineapple fruit vinegar contains more malic acid and citric acid, which related to the characteristics of pineapple, pineapple itself contains a large number of malic acid and citric acid , and in the process of fermentation, malic acid and citric acid remained stable.

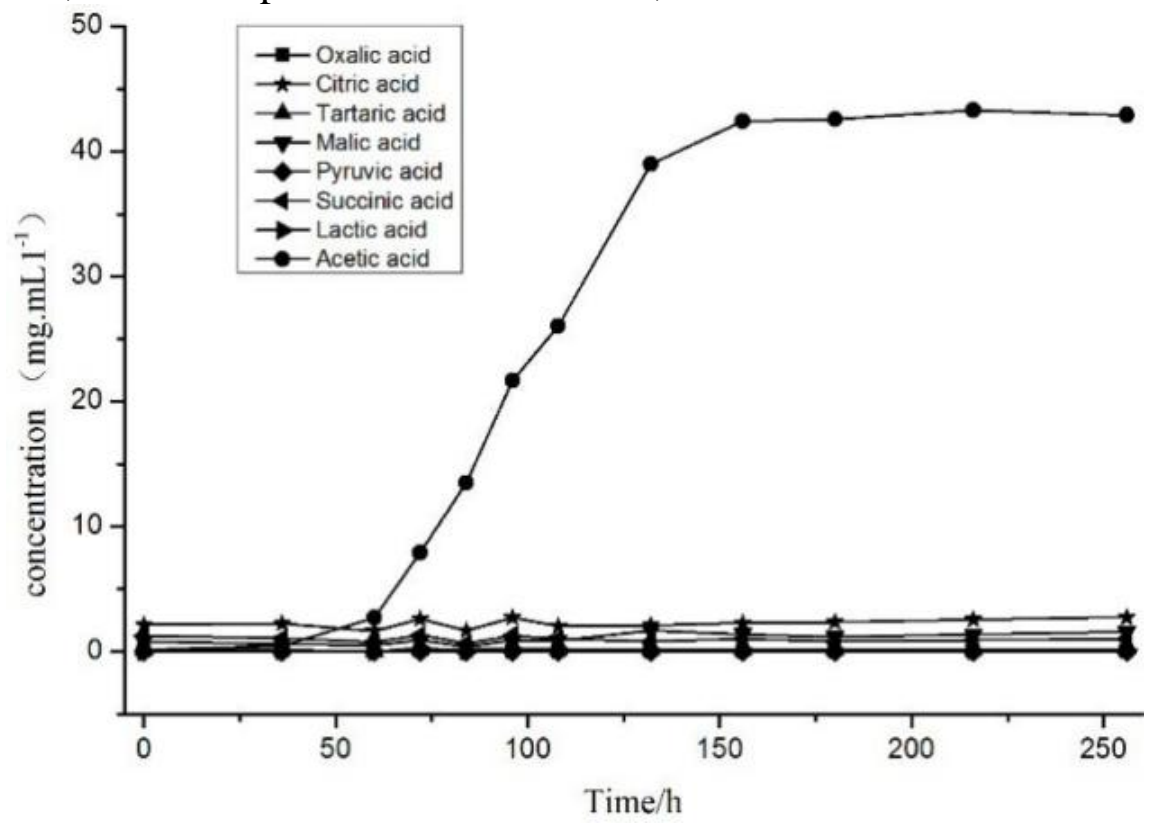

Fig.3 Change of orange acid

\subsection{Polyphenol and antioxidant activity}

The figure 4 and 5 shows that in the process of fermentation, gradually reduce the DPPH clearance from 0 to 5 days, 5 days later, increased clearance; ABTS clearance rate increased during the fermentation process, increased from $65.3 \%$ to $81.6 \%$.The figure 8 shows that in the process of fruit vinegar fermentation, the change of the total reducing power presents similar to DPPH trend, and in $5 \sim 9 \mathrm{~d}$, total reducing power rise rapidly.In this paper, the results DPPH clearance and the total reducing power and zhang qiang hong mei fruit vinegar fermentation process in the study of oxidation resistance change is consistent; Sun Luhong research shows that antioxidant capacity and polyphenols, flavonoids and other closely related material, acetic acid fermentation process, ethanol content gradually reduce, polyphenols and flavonoids of exhalation, solution of antioxidant ability; At the same time, the research shows that antioxidant capacity and total acid showed positive correlation, as the accumulation of acetic acid, antioxidant capacity increased gradually. 


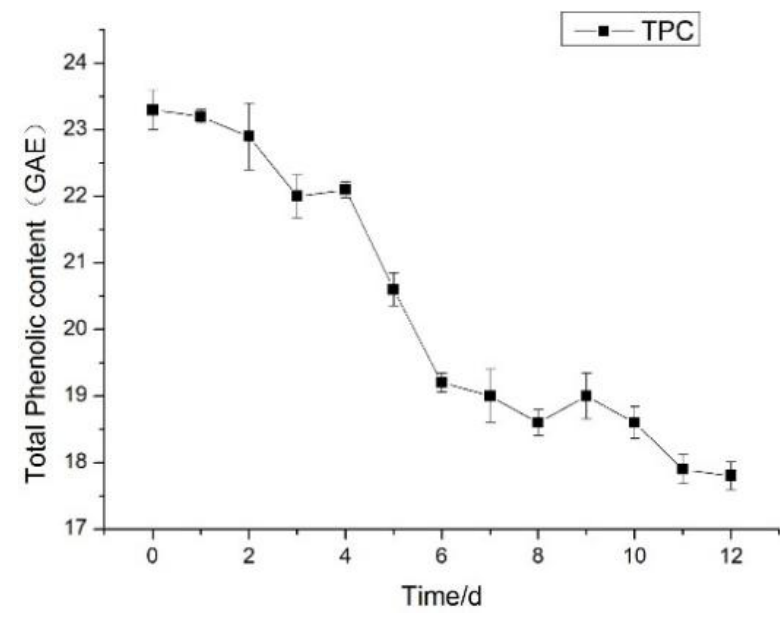

Fig.4 Chang of total phenolic content

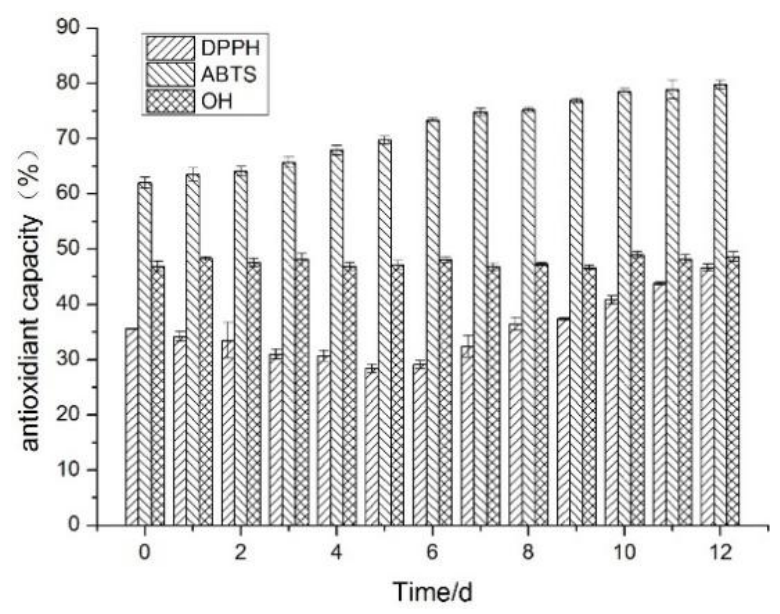

Fig.5 Change of antioxidant capacity

\subsection{HS-SPME-GC-MS analysis of volatile compounds in wine sample}

Table 1 The change of Volatile components during the fermentation of pineapple peel vinegar

\begin{tabular}{|c|c|c|c|c|}
\hline \multirow{2}{*}{ No } & \multirow{2}{*}{ Compounds } & \multirow{2}{*}{ Formula } & \multicolumn{2}{|c|}{ Relative content $/ \%$} \\
\hline & & & Before & After \\
\hline 1 & Ethanol & $\mathrm{C}_{2} \mathrm{H}_{6} \mathrm{O}$ & 16.98 & \\
\hline 2 & 2-Pentanone & $\mathrm{C}_{5} \mathrm{H}_{10} \mathrm{O}$ & 0.62 & \\
\hline 3 & 2,4,5-Trimethyl-1,3-dioxolane & $\mathrm{C}_{6} \mathrm{H}_{12} \mathrm{O}_{2}$ & 2.08 & \\
\hline 4 & BUTANOIC ACID, ETHYL ESTER & $\mathrm{C}_{6} \mathrm{H}_{12} \mathrm{O}_{2}$ & 0.03 & \\
\hline 5 & 1-Propanol, 2-methyl- & $\mathrm{C}_{4} \mathrm{H}_{10} \mathrm{O}$ & 1.96 & \\
\hline 6 & 1-Butanol, 3-methyl- & $\mathrm{C}_{7} \mathrm{H}_{14} \mathrm{O}_{2}$ & 26.31 & \\
\hline 7 & Hexanoic acid, ethyl ester & $\mathrm{C}_{8} \mathrm{H}_{16} \mathrm{O}_{2}$ & 1.15 & \\
\hline 8 & OCTANOIC ACID, ETHYL ESTER & $\mathrm{C}_{10} \mathrm{H}_{20} \mathrm{O}_{2}$ & 8.10 & \\
\hline 9 & Acetic acid & $\mathrm{C}_{2} \mathrm{H}_{4} \mathrm{O}_{2}$ & 0.65 & 52.71 \\
\hline 10 & Propanoic acid, 2-methyl- & $\mathrm{C}_{4} \mathrm{H}_{8} \mathrm{O}_{2}$ & & 1.46 \\
\hline 11 & Decanoic acid, ethyl ester & $\mathrm{C}_{12} \mathrm{H}_{24} \mathrm{O}_{2}$ & 5.21 & \\
\hline 12 & Butanoic acid, 3-methyl- & $\mathrm{C}_{5} \mathrm{H}_{10} \mathrm{O}_{2}$ & 0.42 & 4.39 \\
\hline 13 & BUTANEDIOIC ACID, DIETHYL & $\mathrm{C}_{8} \mathrm{H}_{14} \mathrm{O}_{4}$ & 1.01 & \\
\hline 14 & Acetic acid, 2-phenylethyl ester & $\mathrm{C}_{10} \mathrm{H}_{12} \mathrm{O}_{2}$ & 5.22 & \\
\hline 15 & Hexanoic acid & $\mathrm{C}_{6} \mathrm{H}_{12} \mathrm{O}_{2}$ & 2.75 & 4.43 \\
\hline 16 & Benzeneethanol & $\mathrm{C}_{8} \mathrm{H}_{10} \mathrm{O}$ & 14.05 & 18.73 \\
\hline 17 & Benzeneacetic acid & $\mathrm{C}_{8} \mathrm{H}_{8} \mathrm{O}_{2}$ & & 1.38 \\
\hline 18 & Octanoic acid & $\mathrm{C}_{8} \mathrm{H}_{16} \mathrm{O}_{2}$ & 5.84 & 7.59 \\
\hline 19 & Nonanoic acid & $\mathrm{C}_{9} \mathrm{H}_{18} \mathrm{O}_{2}$ & 0.13 & 0.57 \\
\hline 20 & Decanoic acid & $\mathrm{C}_{10} \mathrm{H}_{20} \mathrm{O}_{2}$ & 0.76 & 0.86 \\
\hline 21 & Benzoic acid & $\mathrm{C}_{7} \mathrm{H}_{6} \mathrm{O}_{2}$ & 0.11 & 1.09 \\
\hline Esters & & & 21.76 & 1.9 \\
\hline Organic acid & & & 10.45 & 74.48 \\
\hline Alcohol & & & 60.35 & 18.86 \\
\hline Other & & & 7.44 & 5.02 \\
\hline
\end{tabular}

As shown in tale 1 and figure 6, before and after fermentation, volatile substances change obviously, and after fermentation, small molecules is significantly reduced before fermentation;The table 1 shows that before fermentation, volatile substances sort is more, the ester material was $21.76 \%$, the organic acid is $10.45 \%$, alcohol is $60.35 \%$;After fermentation, the ester matter, organic acids and alcohols were 1.9, 64.81, 18.86\%, ester substances and alcohols sharply lower in the fermentation process, and organic acids in the fermentation process has increased dramatically.Since most of ester material for volatile flavor substances, in the process of 
fermentation because of moving, plenty of flavor substances volatile, resulting in a decrease of esters content quality;At the same time, in the process of fermentation, alcohol decreased dramatically, alcohols increased, in addition to ethanol was completely oxidation, including propanol, butanol and other alcohols are oxidized into acids substances, shows that not only can vinegar bacteria oxidation of ethanol to acetic acid, can also oxidize propyl alcohol, butyl alcohol, etc for the corresponding acid.By GC/MS results can be seen, phenylethyl alcohol in relatively higher percentage before and after fermentation, and little change before and after shows that vinegar bacteria oxidation of benzene ethanol for the corresponding acid [10].

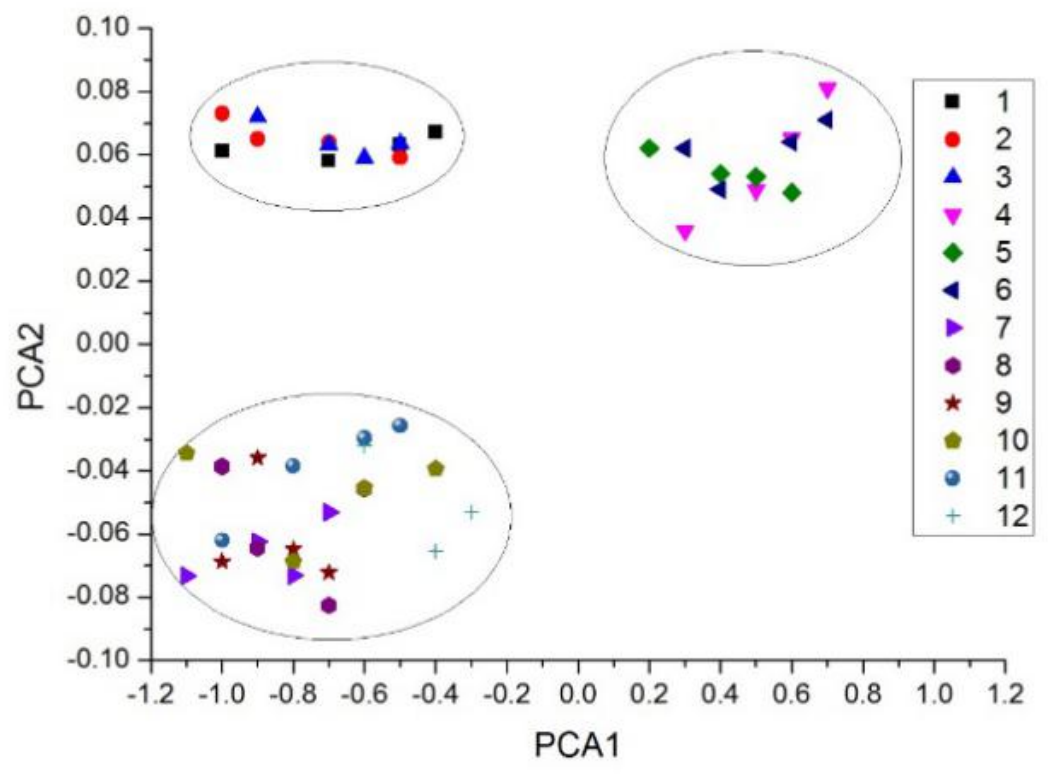

Fig.6 The PCA of volatile compound

\section{Conclusion}

System in this paper to study the pineapple fruit vinegar fermentation and reveals its fermentation characteristics, using strain AS1.41 fruit vinegar fermentation of pineapple peel slag, in $3 \sim 6 \mathrm{~d}$ period fast fermentation, acidity, $\mathrm{pH}$ and alcohol change rapidly during this period, then change is slow; In the fermentation process in acetic acid organic acids change, other classes of organic acid content in a stable state; By comparing the volatile substances change before and after fermentation, the fermentation of volatile substances before and after the change is bigger, mainly alcohols and esters before fermentation, fermentation if left in organic acids and alcohols, esters loss substance content in the fermentation process is more, the fermentation of pineapple fruit vinegar antioxidant ability is fermented before have bigger promotion, the activity of fruit vinegar function was further improved. To be further studied in this paper, we study it is shallow, but also different strains fermentation characteristics and metabolic changes in the process of fermentation, for further understanding of fruit vinegar fermentation process and industrial application.

\section{Acknowledgement}

We gratefully acknowledge financial assistance from Public welfare research and capacity building in Guangdong province special fund project(No:2016A020210005)and China academy of tropical agriculture, basic scientific research business expenses special funds(No:1630122017013). 


\section{References}

[1] Chen Y, Huang Y, Bai Y, et al. Effects of mixed cultures of Saccharomyces cerevisiae and Lactobacillus plantarum in alcoholic fermentation on the physicochemical and sensory properties of citrus vinegar[J]. LWT- Food Science and Technology, 2017, 84.

[2] Ho C W, Lazim A M, Fazry S, et al. Varieties, production, composition and health benefits of vinegars: A review[J]. Food Chemistry, 2016, 221:1621.

[3] Confalonieri U E, Ferreira L F, Araújo A J, et al. Employment of different processes for the production of strawberry vinegars: Effects on antioxidant activity, total phenols and monomeric anthocyanins[J]. LWT - Food Science and Technology, 2013, 52(2):139-145.

[4] Samad A, Azlan A, Ismail A. Therapeutic effects of vinegar: a review[J]. Current Opinion in Food Science, 2016, 8:56-61.

[5] Roda A, Lucini L, Torchio F, et al. Metabolite profiling and volatiles of pineapple wine and vinegar obtained from pineapple waste[J]. Food Chemistry, 2017.

[6] Ubeda C, Callejón R M, Hidalgo C, et al. Determination of major volatile compounds during the production of fruit vinegars by static headspace gas chromatography-mass spectrometry method[J]. Food Research International, 2011, 44(1):259-268.

[7] Zhang X Y, Chen J, Li X L, et al. Dynamic changes in antioxidant activity and biochemical composition of tartary buckwheat leaves during Aspergillus niger, fermentation[J]. Journal of Functional Foods, 2017, 32:375-381.

[8] Chi C H, Cho S J. Improvement of bioactivity of soybean meal by solid-state fermentation with Bacillus amyloliquefaciens, versus Lactobacillus, spp. and Saccharomyces cerevisiae[J]. LWT - Food Science and Technology, 2016, 68:619-625.

[9] Qi Z, Dong D, Yang H, et al. Improving fermented quality of cider vinegar via rational nutrient feeding strategy[J]. Food Chemistry, 2017, 224:312.

[10] Lalou S, Hatzidimitriou E, Papadopoulou M, et al. Beyond traditional balsamic vinegar: Compositional and sensorial characteristics of industrial balsamic vinegars and regulatory requirements[J]. Journal of Food Composition \& Analysis, 2015, 43:175-184. 